\title{
O cuidado em Saúde Mental: contribuições da fisioterapia
}

\author{
Mental Health care: \\ contributions of physiotherapy
}

\section{Fernanda Vicenzi Pavan' ${ }^{1}$ Luisa Verissimo Sampaio ${ }^{2}$ (1) Mônica Weiler Ceccato 3 (1) Suzana Carolina Nascimento 4 (1)}

\begin{abstract}
'Secretaria de Saúde de Timbó. (Timbó). Santa Catarina, Brasil. fevicenzi@gmail.com Escola de Ciências de Saúde e da Vida do Centro Universitário Uniprojeção (Brasília). Distrito Federal, Brasil. ft.luisa.verissimo@gmail.com ${ }^{3}$ Universidade Regional de Blumenau (Blumenau). Santa Catarina, Brasil. monicaweilerceccato@gmail.com ${ }^{4}$ Autora para correspondência. Universidade Federal do ABC (São Bernardo). São Paulo, Brasil. suzanacnascimento23@gmail.com
\end{abstract}

O conceito de saúde mental adotado pela Organização Mundial da Saúde (OMS) relaciona-se ao estado de bem-estar de uma pessoa capaz de realizar suas próprias habilidades, de lidar com as tensões do cotidiano, fazer contribuições à sociedade em que vive. ${ }^{1}$ A saúde mental é parte integrante e essencial da saúde e, portanto, não pode ser abordada em contraposição à saúde física, conforme o equivocado dualismo corpo-mente.

Entende-se que o sofrimento psíquico não se reserva às pessoas que receberam algum diagnóstico específico, mas algo que é presente na vida de todos e que se manifesta de maneira particular a cada um. Por vezes, tais sofrimentos se tornam tão significativos que afetam as atividades sociais, familiares e profissionais.?

Os transtornos mentais são descritos como condições que afetam o funcionamento da cognição, humor, controle de impulsos e comportamento, de modo a comprometer a habilidade do indivíduo no desempenho de suas funções na sociedade, podendo levar a incapacitação, morbidade e morte prematura. Em relação à epidemiologia, estudos brasileiros indicam a prevalência de 30\% de adultos acometidos no período de um ano. $\frac{3}{}$ Belfer ${ }^{4}$ e Kieling et al. .5 afirmam que os transtornos mentais estão entre as dez condições que mais causam incapacidade e vêm sendo cada vez mais discutidos por profissionais do mundo todo.

Dentre as manifestações que caracterizam os transtornos mentais estão alterações na estrutura corporal e no movimento, como dificuldades na realização de movimentos, rigidez muscular, alterações na postura, padrão anormal de respiração e prejuízo da expressão corporal. A presença dessas alterações corporais pode ocorrer em detrimento do próprio transtorno mental ou pela ação prolongada de medicamentos psicotrópicos, esse quadro interfere de maneira significativa na funcionalidade, e consequentemente no cotidiano ${ }^{6}$ das pessoas. 
Dados epidemiológicos demonstram que, em relação ao envelhecimento, as psicopatologias mais comuns são a depressão, ansiedade, demência, esquizofrenia, dependência de álcool e transtornos de personalidade. Acredita-se que esse processo pode estar relacionado com um quadro de vulnerabilidade contribuindo para o desenvolvimento de doenças como os transtornos mentais. Fatores como idade avançada (maior que 80 anos), abandono familiar, solidão, fragilidade social e uso contínuo de cinco ou mais medicamentos também podem influenciar negativamente na funcionalidade do idoso.-12 $\mathrm{A}$ respeito da saúde mental na infância e adolescência, a prevalência pode chegar a $20 \%$ da população nesta faixa etária. Estima-se que conflitos familiares, problemas escolares, bullying, impulsividade e depressão sejam os principais fatores de risco para o suicídio na infância. 13,14

A atenção às pessoas com transtornos mentais, desde a Reforma Psiquiátrica na década de 70, tem como objetivo principal o pleno exercício de sua cidadania, o que implica em oferecer cuidados através de serviços abertos, com participação ativa da comunidade, formando redes com políticas públicas como educação, emprego, moradia dentre outros. ${ }^{2}$ Para Ayres ${ }^{15}$, a relação entre paciente e profissional é sempre um encontro. Este encontro terapêutico deve ser uma verdadeira relação de cuidado. Há na saúde coletiva brasileira uma reconstrução das práticas de saúde que trazem à baila o cuidado em saúde. De acordo com a Política Nacional de Humanização do SUS, o cuidar na saúde é uma dimensão da integralidade que deve permear as práticas de saúde.

Portanto, o cuidado não pertence a um ou outro ofício. Heidegger ${ }^{16}$ considera que o ser do humano é um estar lançado no mundo, numa reconstrução de si mesmo e do mundo e, portanto, pensa ser justo assumir que as práticas de saúde, sejam elas quais forem, e praticadas por quaisquer profissionais de saúde, também se elucidam como cuidado.

Falando especificamente da fisioterapia, esta vem assumindo importância cada vez maior no campo da saúde mental, apresentando-se como opção de tratamento não medicamentoso para a promoção do bem- estar, do autocuidado, da otimização do movimento funcional e da consciência do movimento. ${ }^{17}$ O fisioterapeuta é o profissional de saúde que estuda o movimento do corpo, suas formas de expressão e potencialidades. Está capacitado para atuar na prevenção e tratamento de distúrbios cinéticos funcionais.

Em relação à sua atuação em saúde mental, a fisioterapia pode contribuir para a manutenção do estado de saúde, além de possibilitar a reabilitação da capacidade funcional. Uma das possibilidades de intervenção é através da psicomotricidade, um campo de atuação transdisciplinar cujo objetivo é investigar as relações entre o pensamento e a ação associado às emoções. Na saúde mental, esta prática emprega o corpo e o movimento como instrumento para auxiliar na comunicação social, possibilitar um contato com as emoções e manifestar pensamentos. Além disso, busca melhorar a autoestima e a autoconfiança. 18-20

Duignan, Hedley, Milverton ${ }^{21}$ afirmam que o trabalho do movimento, da consciência corporal, traz diferentes interpretações para as pessoas em sofrimento psíquico, porém, há um consenso de que são grandes os benefícios advindos de sua prática, sejam orgânicos ou psicológicos. Afirma-se que sua ação não é só psicomotora, mas psicossocial. Além disso, a prática corporal terapêutica enquadra-se nos preceitos de saúde, por promover saúde, prevenir doenças e proporcionar a longevidade.

\section{Contribuições das autoras}

Pavan FV, Sampaio LVP, Ceccato MW e Nascimento S participaram da concepção e delineamento do estudo, busca e análise dos dados da pesquisa, interpretação dos resultados, levantamento bibliográfico e redação do artigo científico.

\section{Conflitos de interesses}

Nenhum conflito financeiro, legal ou político envolvendo terceiros (governo, empresas e fundações privadas, etc.) foi declarado para nenhum aspecto do trabalho submetido (incluindo, mas não se limitando a subvenções e financiamentos, participação em conselho consultivo, desenho de estudo, preparação de manuscrito, análise estatística, etc.). 


\section{Referências}

1. Organização mundial da saúde, Organização Panamericana de saúde. OPAS/OMS apoia governos no objetivo de fortalecer e promover a saúde mental da população [Internet]. 2016. Disponível em: https://simepar.org.br/blog/opasoms-apoiagovernos-no-objetivo-de-fortalecer-e-promover-a-saude-mentalda-populacao/

2. Ministério da Saúde (Brasil), Secretaria de Atenção à Saúde. Cadernos de Atenção Básica: Saúde Mental [Internet]. Brasília: Ministério da Saúde; 2013. Disponível em: https://bvsms.saude. gov.br/bvs/publicacoes/cadernos atencao basica 34 saude mental.pdf

3. Andrade LSG, Wang Y. Epidemiologia dos transtornos psiquiátricos. In: Alvarenga PG, Andrade AG. Fundamentos em psiquiatria. Barueri, SP: Manole; 2008. p. 1-12.

4. Belfer ML. Child and adolescent mental disorders: the magnitude of the problem across the globe. J Child Psychol Psychiatry. 2008;49(3):226-36. https://doi.org/10.1111/j.14697610.2007.01855.x

5. Kieling C, Baker-Henningham H, Belfer M, Conti G, Ertem I, Omigbodun O, et al. Child and adolescent mental health worldwide: evidence for action. Lancet. 2011;378(9801):1515-25. https://doi.org/10.1016/s0140-6736(11)60827-1

6. Silva SB, Pedrão LJ, Miasso Al. O impacto da fisioterapia na reabilitação psicossocial de portadores de transtornos mentais. SMAD, Rev. Eletrônica Saúde Mental Álcool Drog. [Internet]. 2012;8(1):34-40. Disponível em: http://pepsic.bvsalud.org/scielo. php?script=sci abstract\&pid=S1806-69762012000100006

7. Caixeta L, Vieira RT. Epidemiologia dos transtornos mentais em idosos. In: Busse EW, Blazer DG. Psiquiatria Geriátrica. Porto Alegre: Artmed; 2016. p. 32-50.

8. Gullich I, Duro SMS, Cesar JA. Depressão entre idosos: um estudo de base populacional no Sul do Brasil. Rev. bras. epidemiol. 2016;19(4):691-701. https://doi.org/10.1590/1980$\underline{5497201600040001}$

9. Ramos FP, Silva SC, Freitas DF, Gangussu LM, Bicalho AH, Sousa BVO, et al. Fatores associados à depressão em idoso. Revista Eletrônica Acervo Saúde. [Internet]. 2019;(19):e239. Disponível em: https://www.acervomais.com.br/index.php/saude/article/ view/239/154

10. Forner FC, Alves CF. Uma revisão de literatura sobre os fatores que contribuem para o envelhecimento ativo na atualidade. Revista Universo Psi [Internet]. 2019;1(1):150-74. Disponível em: https://seer.faccat.br/index.php/psi/article/view/1344
11. Melo MG, Santos RP, Tavares SS, Saraiva APC, Carvalho AKN, Pereira JHR, et al. Aplicação da escala de depressão geriátrica abreviada em idosos ativos e sedentários do HIPERDIA. Revista Eletrônica Acervo Saúde [Internet]. 2020;12(9):e4214. Disponível em: https://www.acervomais.com.br/index.php/saude/article/ view/4214/2707

12. Costa SMM, Ramos FCN, Barbosa E, Santos NB. Aspectos sociais das relações entre a depressão e o isolamento dos idosos. GIGAPP Estudios Working Papers [Internet]. 2020;7(155):292-308. Disponível em: http://www.gigapp.org/ewp/index.php/GIGAPPEWP/article/view/187/202

13. Sousa GS, Santos MSP, Silva ATP, Perrelli JGA, Sougey EB. Revisão de literatura sobre suicídio na infância. Ciênc. saúde coletiva. 2017;22(9):3099-110. http://dx.doi.org/10.1590/141381232017229.14582017

14. Almeida RS, Lima RC, Crenzel G, Abranches CD. Epidemiologia dos transtornos mentais da infância e adolescência. Saúde mental da criança e do adolescente. $2^{\mathrm{a}}$ ed. Barueri: Manole; 2019. p. 30-6.

15. Ayres JRCM. O cuidado, os modos de ser (do) humano e as práticas de saúde. Saúde soc. 2004;13(3):16-29. https://doi. org/10.1590/S0104-12902004000300003

16. Heidegger M. Ser e Tempo. Petrópolis: Vozes; 1995.

17. Probst M. Physiotherapy and mental health. In: Clinical Physical Therapy [Internet]. Londres: InTechOpen; 2017. Disponível em: https://www.intechopen.com/books/clinicalphysical-therapy/physiotherapy-and-mental-health

18. Costa MLA, Lima DC, Lucena MM, Oliveira VD. Psicomotricidade como instrumento da fisioterapia na saúde mental. In: Daltro MCSL, Garcia VVCG. Fisioterapia na Saúde Mental. Patos: FIP; 2016. p.41-66.

19. Conselho Regional de Fisioterapia e Terapia Ocupacional da $11^{\text {a }}$ Região. Fisioterapia: definições [Internet]. Brasília. Disponível em: https://crefito11.gov.br/definicoes-da-fisioterapia.php

20. Conselho Regional de Fisioterapia e Terapia Ocupacional da Primeira Região (Brasil). Saúde Mental: Atuação da fisioterapia e da terapia ocupacional [Internet]. Recife: Crefito1; 2017. Disponível em: http://www.crefito1.org.br/imagens/revistas/ CARTILHA_SAUDE_MENTAL_WEB-1.pdf

21. Duignan D, Hedley L, Milverton R. Exploring dance as a therapy for symptoms and social interaction in a dementia care unit. Nurs Times. 2009;105(30):19-22. Citado em: PMID: 19736794 